\title{
Terahertz vortex wave generation in air plasma by two-color femtosecond laser pulses
}

\author{
Maksym IVAnov ${ }^{1, *}$, Illia Thiele ${ }^{2}$, Luc Bergé 3 , Stefan SKupin ${ }^{4}$, Danas Buožius ${ }^{1}$, ANd \\ VIRGILIJUS VAIČAITIS ${ }^{1}$ \\ ${ }^{1}$ Laser Research Center, Vilnius University, 10 Sauletekio avenue, Vilnius, Lithuania, LT-10223 \\ ${ }^{2}$ Department of Physics, Chalmers University of Technology, Göteborg, Sweden, SE-412 96 \\ ${ }^{3}$ CEA, DAM, Arpajon, France, DIF - 91297 \\ ${ }^{4}$ Institut Lumiere Matiere, UMR 5306 Université Lyon 1 - CNRS, Université de Lyon, Villeurbanne, France, F-69622 \\ *Corresponding author: maks.ivannov@gmail.com \\ Compiled June 17, 2019
}

We investigate the generation of broadband terahertz (THz) pulses with phase singularity from air plasmas created by fundamental and second harmonic laser pulses. We show that when the second harmonic beam carries a vortex charge, the $\mathrm{THz}$ beam acquires a vortex structure as well. A generic feature of such $\mathrm{THz}$ vortex is that the intensity is modulated along the azimuthal angle, which can be attributed to the spatially varying relative phase difference between the two pump harmonics. Fully space and time resolved numerical simulations reveal that transverse instabilities of the pump further affect the emitted $\mathrm{THz}$ field along nonlinear propagation, which produces additional singularities resulting in a rich vortex structure. The predicted intensity modulation is experimentally demonstrated with a thermal camera, in excellent agreement with simulation results. The presence of phase singularities in the experiment is revealed by astigmatic transformation of the beam using a cylindrical mirror. (๑) 2019 Optical Society of America

http://dx.doi.org/10.1364/ao.XX.XXXXXX

\section{INTRODUCTION}

Terahertz $(\mathrm{THz})$ radiation is of great current interest due to many applications such as nonlinear $\mathrm{THz}$ spectroscopy and imaging [1] or electron bunch compression [2]. A compact and effective method to obtain very high $\mathrm{THz}$ field strengths and extremely broadband spectral widths is $\mathrm{THz}$ wave generation from plasma filaments formed in air by focused bichromatic femtosecond laser pulses consisting of first harmonic (FH) and second harmonic (SH) waves [3-6]. On the other hand, special light fields, such as optical vortex [7], radially polarized [8], Bessel $[9,10]$ and Airy beams [11] are widely studied and employed in various fields $[12,13]$. Previous attempts on vortex generation at $\mathrm{THz}$ frequencies [14-21] were exclusively based on manipulation of $\mathrm{THz}$ waves by employing external components which are inherently limited in terms of acceptable bandwidth. Therefore, alternative methods for ultra-broadband vortex generation at $\mathrm{THz}$ frequencies should be proposed and investigated. Very recently, vortex-shaped $\mathrm{THz}$ pulses have been already generated without external shaping elements [22, 23] in ZnTe crystal, which, however, supply relatively narrow bandwidths.

In this work we investigate vortex $\mathrm{THz}$ pulse generation in an air-plasma induced by the coupling between Gaussian $\mathrm{FH}$ and vortex $\mathrm{SH}$ pulses. In this novel scheme, the vorticity is created already at the $\mathrm{THz}$ generation stage. It appears that SH carrying an optical vortex charge affects not only the phase but also the intensity distribution of the generated $\mathrm{THz}$ pulse. We distinguish two stages of $\mathrm{THz}$ vortex generation: (i) At the beginning of the plasma filament, an intensity modulated $\mathrm{THz}$ vortex pulse is created. This intensity modulation is frequency dependent. (ii) Upon further propagation, the pump pulse may undergo spatio-temporal instabilities which induce secondary phase singularities in the $\mathrm{THz}$ field, but the total topological charge is conserved. Results of our investigation suggest an alternative method for the generation of structured $\mathrm{THz}$ waves spanning ultrabroadband frequency ranges.

\section{THEORY}

For two color-laser-induced gas plasmas, the ionization current mechanism [24] is the key player for THz emission. This emission is caused by the macroscopic current of free electrons which are created by field ionization in the tunneling regime $[25,26]$ and driven by the laser electric field. The principal electric field component is transverse to the laser propagation direction, and we consider linear polarization. Neglecting electron collisions, the current equation reads

$$
\partial_{t} J=\frac{q_{\mathrm{e}}^{2}}{m_{\mathrm{e}}} n_{\mathrm{e}} E
$$

with electron charge $q_{\mathrm{e}}$, mass $m_{\mathrm{e}}$, electron density $n_{\mathrm{e}}$ and electric field $E$. The electron density is a time-dependent parameter governed by the ionization rate and can be computed using ionization rate equations $[5,27]$. The electric field consists of both optical frequency components and generated $\mathrm{THz}$ components, which plays an important role in the $\mathrm{THz}$ spectral broadening [28]. However, certain effects can be already understood by 

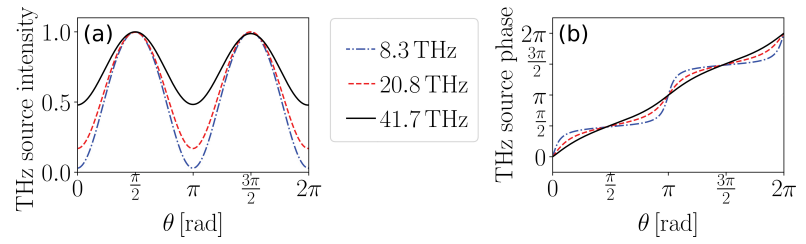

Fig. 1. Azimuthal intensity (a) and phase (b) distribution of the Fourier-transformed THz source term $\partial_{t} J$ [see Eqs. (1), (2)].

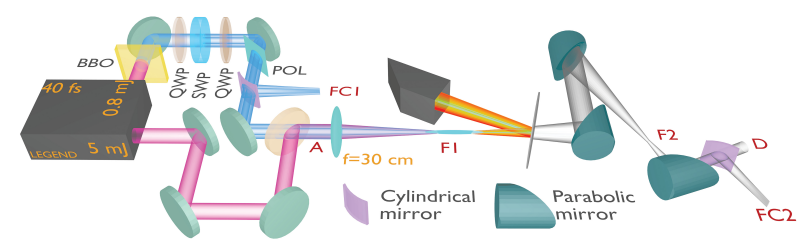

Fig. 2. Sketch of experimental setup. QWP - quarter wave plate, SWP - S-waveplate (q-plate), POL - polarizer, BBO - nonlinear crystal for $\mathrm{SH}$ generation. $\mathrm{SH}$ is shown by blue, $\mathrm{FH}$ by red-pink, and $\mathrm{THz}$ radiation by gray color. Red letters label relevant positions (see text for details). THz fluency profiles taken in positions 'D' and 'FC2' are shown in Fig. 3 and 4.

considering only the impact of the bichromatic laser electric field. The down-conversion from laser to $\mathrm{THz}$ frequencies takes place because of the nonlinear product between the electron density and the laser electric field. It can be shown that at least two laser colors, here $\mathrm{FH}$ and $\mathrm{SH}$ with a relative phase angle of $\pi / 2$, are required to obtain an efficient down-conversion towards $\mathrm{THz}$ frequencies $[6,29]$. In order to understand this down-conversion process in the case of vortex pump pulses, we first evaluate Eq. (1) for the real-valued laser electric field

$$
E_{\mathrm{L}}(t, \theta)=A(t)\left[E_{\omega_{\mathrm{L}}} \cos \left(\omega_{\mathrm{L}} t\right)+E_{2 \omega_{\mathrm{L}}} \cos \left(2 \omega_{\mathrm{L}} t+\phi+l_{\mathrm{SH}} \theta\right)\right]
$$

with fundamental frequency $\omega_{\mathrm{L}}$, relative phase offset $\phi$, pulse envelope $A(t) ; l_{\mathrm{SH}}$ is the vortex charge of the SH beam. Here, we omit the radial and longitudinal coordinates, and write the electric field as a function of time $t$ and azimuthal angle $\theta$ only. Equation (2) thus represents the rapid time- and $\theta$-dependent distribution of the electric field along the vortex ring, assuming constant $\mathrm{FH}$ and $\mathrm{SH}$ amplitudes $E_{\omega_{\mathrm{L}}, 2 \omega_{\mathrm{L}}}$. The resulting spectral intensity and phase of the source term $\partial_{t} J$ obtained by Fourier transform is presented in Fig. 1 for $\phi=0$ and $l_{\mathrm{SH}}=1$. The intensity is modulated along $\theta$ and is maximal when the relative phase angle $\left(\phi+l_{\mathrm{SH}} \theta\right)$ between the $\mathrm{SH}$ and $\mathrm{FH}$ takes the values $\pi / 2$ and $3 \pi / 2$ and minimal for 0 and $\pi$ (Fig. 1). The modulation depth is the largest at lower frequencies and decreases for larger frequencies, where also the phase approaches a linear ramp-up along $\theta$ as expected for a vortex. The results from this simple theoretical approach remain qualitatively the same when changing the laser and gas parameters within the parameter range allowing for efficient $\mathrm{THz}$ generation.

\section{EXPERIMENTS}

The experimental setup is sketched in Fig. 2. We used a $1 \mathrm{kHz}$ repetition rate laser system (Legend elite duo $\mathrm{HE}+$, Coherent Inc.). Right before the main focusing lens (silica, $f=30 \mathrm{~cm}$ ) labeled 'A' in the setup, the FH pulse had central wavelength of $790 \mathrm{~nm}$, FWHM pulse duration $50 \mathrm{fs}$ and energy of $6 \mathrm{~mJ}$. The SH pulse had central wavelength of $395 \mathrm{~nm}$, FWHM duration $\sim 50 \mathrm{fs}$

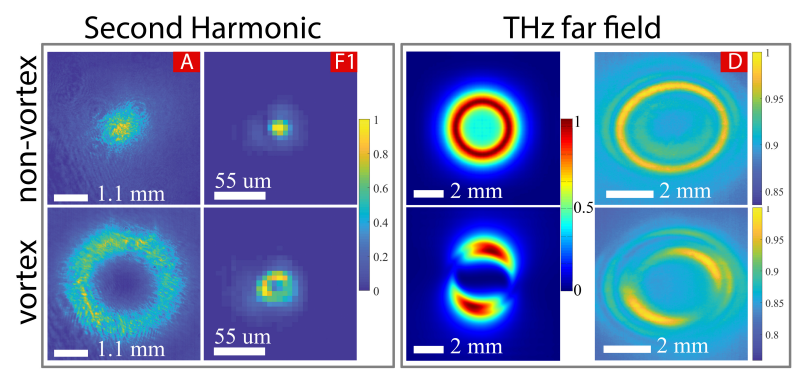

Fig. 3. (Left panel) Images of second harmonic fluency (nonvortex and vortex, for Gaussian and vortex state, respectively). Column labeling correspond to locations where images were acquired as specified in Fig. 2. (Right panel) Corresponding simulated (first column) and experimentally observed (second column) farfield $\mathrm{THz}$ fluencies. See text for details.

and energy of $60 \mu \mathrm{J}$ in Gaussian state and $50 \mu \mathrm{J}$ in vortex state. Our Gaussian SH pulse was shaped into an optical vortex by the method described in [30], which ensures more than one-octave spectral bandwidth of vortex generation: First $\mathrm{SH}$ was circularly polarized by a quarter wave plate (QWP) and converted to an optical vortex (OV) beam by an S-waveplate (RPC-405-06557, Workshop of Photonics) (SWP). Subsequent polarization filtering by a second quarter wave plate (QWP) and a polarizer (POL) ensured generation of a linearly polarized vortex in the $\mathrm{SH}$ beam profile over the whole spectral bandwidth of the $\mathrm{SH}$ pulse. A $0.5 \mathrm{~mm}$ thick $\mathrm{Si}$ wafer and various commercial $\mathrm{THz}$ filters were used to remove the high frequency part of the pump and transmit only $\mathrm{THz}$ radiation, which was then collimated and shrank by parabolic mirrors in a telescope configuration to match the detector aperture. Imaging of the generated $\mathrm{THz}$ beam was performed with a thermal camera detector (VarioCAM head HiRes 640, InfraTec $\mathrm{GmbH}$ ), sensitive in the range $0.1-40 \mathrm{THz}(3000-7.5 \mu \mathrm{m})$. Spectra of $\mathrm{THz}$ radiation were obtained from Fourier transformed interferometric measurements using pyroelectric detector (TPR-A-65 THz, Spectrum Detector Inc.), sensitive in the range $0.1-300 \mathrm{THz}(3000-1 \mu \mathrm{m})$ with a flat response function from $\sim 3$ to $\sim 100 \mathrm{THz}$. The laser-to- $\mathrm{THz}$ conversion efficiency for the regular Gaussian pulses was about $10^{-4}$, but dropped to $\sim 10^{-5}$ in the case of the SH vortex pump, which we attribute to the differences in the spatial intensity distribution of the Gaussian FH and vortex SH beam.

During the experiment the FH beam was always kept Gaussian. Experimental images of the $\mathrm{SH}$ beam are shown in the left panel of Fig. 3. The first and the second row present the $\mathrm{SH}$ Gaussian ('non-vortex') and vortex beam ('vortex'), respectively. The first column 'A' shows images of the harmonics prior to the main focusing lens at position A (see Fig. 2); the second column 'F1' refers to the focus position (F1 in Fig. 2). The FWHM beam widths are 33 and $38 \mu \mathrm{m}$ for the $\mathrm{FH}$ and the vortex $\mathrm{SH}$ in the focal plane of the $30-\mathrm{cm}$ focusing lens, respectively.

Experimental images of the $\mathrm{THz}$ farfield fluency obtained in position ' $\mathrm{D}$ ' (Fig. 2) with the thermal camera (0.1-40 THz) are shown in the last column of Fig. 3). The THz fluency obtained with Gaussian SH (upper row) has a symmetric ring intensity distribution, as expected for conical THz emission [31, 32]. In contrast, the $\mathrm{THz}$ fluency obtained with vortex $\mathrm{SH}$ (lower row) has an intensity modulation manifesting as two maxima along the azimuthal angle, in agreement with our theoretical predictions (see Fig. 1). Simulation results shown for comparison in the first column of right-hand panel in Fig. 3) are in excellent 


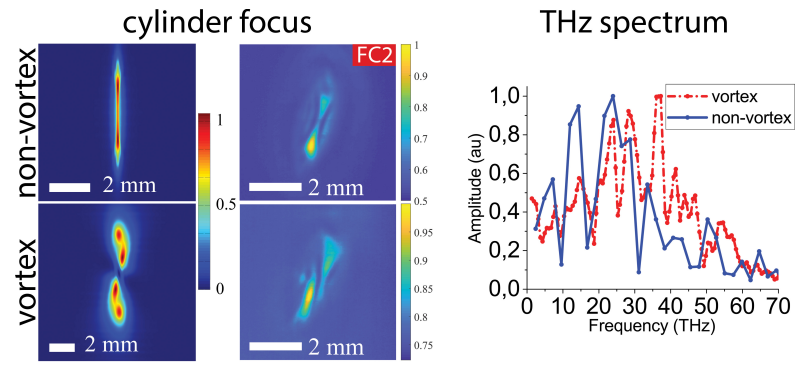

Fig. 4. (Left panel) Simulated (first column) and experimentally observed (second column) fluencies of the generated $\mathrm{THz}$ beams in the focus of a cylindrical mirror $(\mathrm{f}=20 \mathrm{~cm})$. The right-hand side panel shows typical experimental amplitude spectra of the generated $\mathrm{THz}$ pulses in the case of vortex $\mathrm{SH}$ (red dash-dot "vortex") and Gaussian SH (blue solid line "nonvortex").

agreement. The simulated fluency is obtained for the spectral range of 0.01 to $50 \mathrm{THz}$ (see below for details on the simulations).

The presence of a phase singularity in the generated $\mathrm{THz}$ beam was confirmed by the well-controlled method of topological charge determination based on the astigmatic transformation of singular beams by a cylindrical mirror [33]. This method is valid even in the presence of intensity modulations affecting the generated $\mathrm{THz}$ beam profile. A beam without phase singularity focuses in a single line, whereas the appearance of a dark stripe in this line indicates a singular phase. The number of lines and their tilt correspond to the value and sign of the topological charge. As shown in the left panel of Fig. 4, the single dark stripe in the second row suggests that the topological charge of the generated $\mathrm{THz}$ vortex is $\left|l_{T H z}\right|=1$. Simulation results and experimental images show again excellent agreement.

As evidenced by the right-hand panel of Fig. 4, the experimental spectra of the generated $\mathrm{THz}$ pulses are broadband, spanning from 10 to $40 \mathrm{THz}$. We attribute the dips in these spectra to absorbance in optical elements such as Si filters and pellicle beam splitters. In the case of the $\mathrm{THz}$ vortex the peak spectral intensity is located around $35 \mathrm{THz}$, while $\mathrm{THz}$ pulses without phase singularity have their maximum around $25 \mathrm{THz}$. This shift may occur because of the lower ionization rate at the periphery of the $\mathrm{FH}$ pump beam where the $\mathrm{SH}$ vortex has maximum intensity and due to transverse phase variations.

An astigmatic transformation of the generated vortex $\mathrm{THz}$ beam by a cylindrical mirror was used as the primary experimental detection method of the phase singularity, yielding patterns in excellent agreement with our simulation results. However, we also looked at alternative methods to confirm that our understanding of intensity modulated $\mathrm{THz}$ vortex production is indeed correct. According to our theory, the azimuthal intensity modulation comes from the fact that the phase of the SH vortex beam changes by $2 \pi$ over the full azimuthal angle, while the phase of the $\mathrm{FH}$ is constant along this angle. Thus, changing the relative phase between $\mathrm{FH}$ Gaussian and $\mathrm{SH}$ vortex should result in a rotation of the $\mathrm{THz}$ vortex intensity modulation. To verify this property, the $\mathrm{THz}$ vortex intensity distribution was recorded by the thermal camera over several minutes (not shown). The relative phase between $\mathrm{FH}$ and $\mathrm{SH}$ was not under control and fluctuated due to long beam pass distances ( $>5$ meters for each harmonic), vibrations in the room/building, temperature variations, etc. Because of the random nature of the fluctuations, the $\mathrm{THz}$ vortex intensity distribution clearly jittered and made

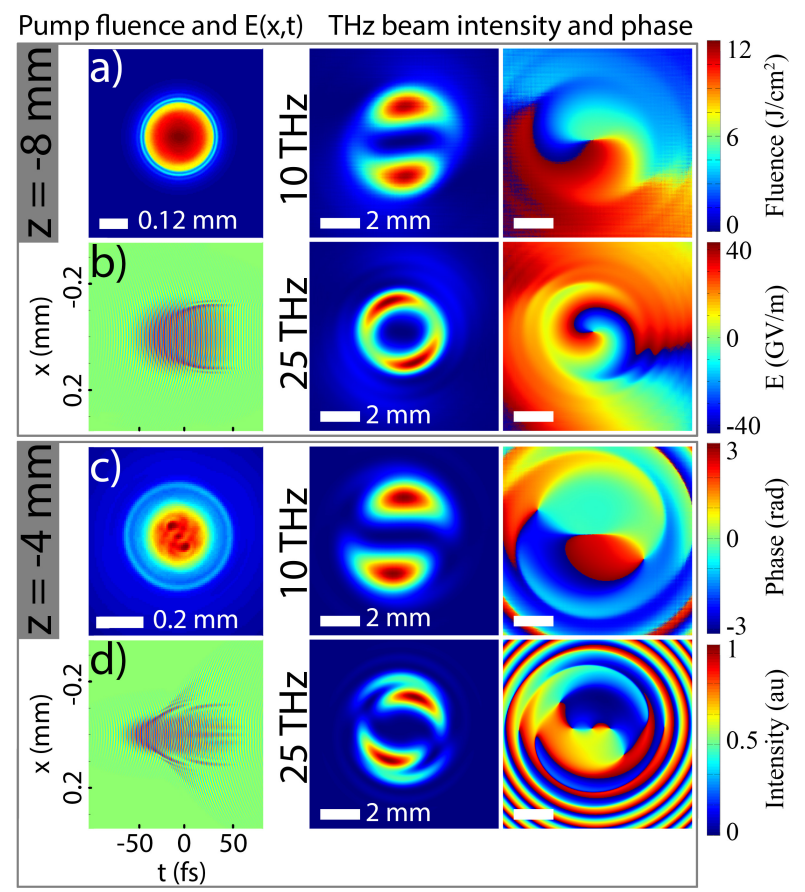

Fig. 5. (a) and (c) show fluency, (b) and (d) show electric field $E(x, t)$ of the two-color pump at the beginning of the filament $(z=-8 \mathrm{~mm})$ and after transverse pump instabilities have fully developed $(z=-4 \mathrm{~mm})$. The two right-hand side columns show collimated far field intensity and phase of the 10 and $25 \mathrm{THz}$ frequency components computed from the field at $z=-8 \mathrm{~mm}$ and $z=-4 \mathrm{~mm}$, respectively (see text for details). Linear focus F1 is at $z=0$ (see Fig. 2).

even a full rotation once in a while. In contrast, when we used $\mathrm{FH}$ and SH Gaussian beam profiles, the intensity distribution of the $\mathrm{THz}$ beam (without phase singularity) did not change. The maximum of the $\mathrm{THz}$ beam just slightly oscillated around the same position due to (flaws in) the pointing stability of the laser.

\section{SIMULATIONS}

For a complementary analysis we performed comprehensive numerical simulations of the full experiment by means of a unidirectional pulse propagation solver $[6,34]$. Laser parameters and focusing conditions in the simulations are chosen such as in the experiments. Our simulation results reveal two distinct stages in the formation and subsequent evolution of the generated $\mathrm{THz}$ pulses. First, intensity and phase modulations of the $\mathrm{THz}$ source term due to the spatially variable relative phase difference between $\mathrm{FH}$ and vortex $\mathrm{SH}$ pump components emerge, as expected from our theoretical predictions (see Fig. 1). Second, the pump pulse develops spatio-temporal instabilities generic to the filamentation dynamics that directly affect the $\mathrm{THz}$ pulse distribution.

The right hand side of Fig. 5 shows collimated far field intensities and phases of the generated 10 and $25 \mathrm{THz}$ spectral components at two stages of the propagation, namely at the beginning of the plasma filament $(z=-8 \mathrm{~mm})$, and after transverse pump instabilities have fully developed $(z=-4 \mathrm{~mm})$. All $z$ positions are given relative to the focus position F1 $(z=0 \mathrm{~mm})$ of the main pump focusing lens (see Fig. 2). To obtain the $\mathrm{THz}$ far field, the nonlinear interaction was stopped at the given $z$ 
position and the THz field was further propagated over a few centimeters in vacuum (sufficiently to reach the far field) and collimated with a lens in thin element approximation (quadratic phase).

At the beginning of the filament $(z=-8 \mathrm{~mm})$ the pump fluency is uniform, as evidenced by Fig. 5 (a). The corresponding spatio-temporal electric field profile in Fig. 5 (b) shows that plasma has only started to deplete the trailing part of the pump pulse, as one would expect from the usual filamentation dynamics $[34,35]$. The generated $\mathrm{THz}$ vortex at this early stage of pump propagation has a single phase singularity in the center. The intensity is circular with azimuthal modulation, and the modulation depth is larger for lower frequencies, as predicted by Fig. 1 . In contrast, at $(z=-4 \mathrm{~mm})$ the pump pulse has undergone severe transverse distortion [see fluency in Fig. 5 (c)], and the spatio-temporal field profile in Fig. 5 (d) looks much more complex. These strong perturbations of the pump clearly affect the intensity distribution of the $\mathrm{THz}$ vortex and produce secondary singularities in the generated $\mathrm{THz}$ field. Nevertheless, additional singularities have alternating signs such that the total topological charge of the singular $\mathrm{THz}\left|l_{T H z}\right|=1$ is preserved during propagation at all relevant frequency components. Overall, our simulations results suggest that the produced $\mathrm{THz}$ vortices are surprisingly stable against pump distortions.

\section{CONCLUSIONS}

We have investigated the properties of $\mathrm{THz}$ radiation generated in air plasma by focused bichromatic femtosecond laser pulses, when one of the pump beams (second harmonic) is an optical vortex. The presence of a phase singularity in the generated $\mathrm{THz}$ beam was confirmed by astigmatic transformation of the singular $\mathrm{THz}$ beams in the focus of a cylindrical lens, as well as by fully space and time resolved numerical simulations. We report that, in contrast to other nonlinear processes (second harmonic generation, parametric generation, etc.), the $\mathrm{THz}$ radiation generated by electron currents in a plasma filament can not be characterized as a THz vortex beam in the 'classical' sense, such as a pure Laguerre-Gaussian beam. Instead, the intensity of the THz beam is modulated along the beam azimuthal angle and contains two minima between two lobes of maximum intensity. This is because the relative phase between two harmonics varies azimuthally when the SH pump pulse is a vortex. Moreover, our numerical simulations demonstrate that transverse instabilities in the filamentary pump propagation affect the $\mathrm{THz}$ vortex without destroying it. They may introduce secondary phase singularities, which renders the phase topology of produced structured $\mathrm{THz}$ fields particularly rich. One of the benefits of $\mathrm{THz}$ generation from plasma currents is the large ( $>40 \mathrm{THz}$ ) spectral range achievable, contrary to bandwidth limited external $\mathrm{THz}$ shaping techniques. We envisage that different combinations of the topological charges of the $\mathrm{FH}$ and $\mathrm{SH}$ pulses open a wide playground for the creation of structured singular THz sources.

Funding. Laserlab-Europe EU-H2020 654148. This research was funded by a grant (No. S-MIP-19-46) from the Research Council of Lithuania. Simulations were performed using HPC resources from Grand Équipement National De Calcul Intensif (Grant No. A0060507594).

Acknowledgments. Authors are grateful to Titas Gertus from Workshop of Photonics, Vilnius, Lithuania, for the Swaveplate used in this experiment as an optical vortex generator.

\section{REFERENCES}

1. P. U. Jepsen, D. G. Cooke, and M. Koch, Laser Photonics Rev 5 (1), 124 (2011).

2. C. Kealhofer, W. Schneider, D. Ehberger, A. Ryabov, F. Krausz, and P. Baum, Science 352, 429 (2016)

3. D. J. Cook and R. M. Hochstrasser, Opt. Lett. 25(16), 1210 (2000).

4. J. D. B. Clough and X.-C. Zhang, Mater. Today 15(1-2), 50 (2012).

5. M. D. Thomson, M. Kress, T. Löffler, and H. G. Roskos, Laser \& Photonics Rev. 1, 349 (2007).

6. I. Babushkin, W. Kuehn, C. Köhler, S. Skupin, L. Berge, K. Reimann, M. Woerner, J. Herrmann, , and T. Elsaesser, Phys. Rev. Lett. 105, 053903 (2010).

7. W. Ji, C.-H. Lee, P. Chen, W. Hu, Y. Ming, L. Zhang, T.-H. Lin, V. Chigrinov, and Y.-Q. Lu, Sci. Reports 6 (2016).

8. P. Stanislovaitis, M. Ivanov, A. Matijosius, V. Smilgevicius, and T. Gertus, Opt. Eng. 56, 095101 (2017).

9. J. Durnin, J. Opt. Soc. Am. A 4(4), 651 (1987).

10. V. Vaicaitis and S. Paulikas, Opt. Quantum Electron. 35(11), 1065 (2003).

11. G. Siviloglou and D. Christodoulides, Opt. Lett. 32(8), 979 (2007).

12. S. W. Hell and J. Wichmann, Opt. Lett. 19, 780 (1994).

13. M. Ivanov and D. Hanstorp, Opt. Commun. 427, 152 (2018).

14. J. He, X. Wang, D. Hu, J. Ye, S. Feng, Q. Kan, and Y. Zhang, Opt. Express 21, 20230 (2013).

15. R. Imai, N. Kanda, T. Higuchi, K. Konishi, and M. Kuwata-Gonokami, Opt. Lett. 39, 3714 (2014).

16. K. Miyamoto, B. J. Kang, W. T. Kim, Y. Sasaki, H. Niinomi, K. Suizu, F. Rotermund, and T. Omatsu, Sci. Reports 6, 38880 (2016).

17. S. Ge, P. Chen, Z. Shen, W. Sun, X. Wang, W. Hu, Y. Zhang, and Y. Lu, Opt. Express 25, 12349 (2017).

18. S.-J. Ge, Z.-X. Shen, P. Chen, X. Liang, X.-K. Wang, W. Hu, Y. Zhang, and Y.-Q. Lu, Crystals 10, 314 (2017).

19. A. Minasyan, C. Trovato, J. Degert, E. Freysz, E. Brasselet, and E. Abraham, Opt. Lett. 42, 41 (2017).

20. C. Liu, J. Liu, L. Niu, X. Wei, K. Wang, and Z. Yang, Sci. Reports 7 , 3891 (2017)

21. Z. Wu, X. Wang, W. Sun, S. Feng, P. Han, J. Ye, Y. Yu, and Y. Zhang, Opt. Express 26, 1506 (2018).

22. Q. Lin, S. Zheng, Q. Song, X. Zeng, Y. Cai, Y. Li, Z. Chen, L. Zha, X. Pan, and S. Xu, Opt. Lett. 44, 887 (2019).

23. A. A. Dhaybi, J. Degert, E. Brasselet, E. Abraham, and E. Freysz, J. Opt. Soc. Am. B 36, 12 (2019).

24. K. Y. Kim, A. J. Taylor, S. L. Chin, and G. Rodriguez, Nat. Photon. 2, 605 (2008).

25. M. Ammosov, N. Delone, and V. Krainov, Sov. Phys. JETP 64, 1191 (1986).

26. G. L. Yudin and M. Y. Ivanov, Phys. Rev. A 64, 013409 (2001).

27. I. Thiele, B. Zhou, A. Nguyen, E. Smetanina, R. Nuter, K. J. Kaltenecker, P. G. de Alaiza Martínez, J. Déchard, L. Bergé, P. U. Jepsen, and S. Skupin, Optica 5, 1617 (2018).

28. E. Cabrera-Granado, Y. Chen, I. Babushkin, L. Bergé, and S. Skupin, New J. Phys. 17, 023060 (2015).

29. K.-Y. Kim, J. H. Glownia, A. J. Taylor, and G. Rodriguez, Opt. Express 15, 4577 (2007).

30. M. Gecevicius, M. Ivanov, M. Beresna, A. Matijosius, V. Tamuliene, T. Gertus, A. Cerkauskaite, K. Redeckas, M. Vengris, V. Smilgevicius, and P. G. Kazansky, J. Opt. Soc. Am. B 35, 190 (2018).

31. P. Klarskov, A. C. Strikwerda, K. Iwaszczuk, and P. U. Jepsen, New J. Phys. 15, 075012 (2013).

32. V. Vaicaitis, M. Ivanov, K. Adomavicius, Z. Svirskas, U. Morgner, and I. Babushkin, Laser Phys. 28, 095402 (2018).

33. V. Denisenko, V. Shvedov, A. S. Desyatnikov, D. N. Neshev, W. Krolikowski, A. Volyar, M. Soskin, and Y. S. Kivshar, Opt. Express 17, 23374 (2009).

34. M. Kolesik and J. V. Moloney, Phys. Rev. E 70, 036604 (2004).

35. A. Vinçotte and L. Bergé, Phys. Rev. Lett. 95, 193901 (2005). 


\section{FULL REFERENCES}

1. P. U. Jepsen, D. G. Cooke, and M. Koch, "Terahertz spectroscopy and imaging - modern techniques and applications," Laser Photonics Rev 5 (1), 124-166 (2011).

2. C. Kealhofer, W. Schneider, D. Ehberger, A. Ryabov, F. Krausz, and P. Baum, "All-optical control and metrology of electron pulses," Science 352, 429-433 (2016).

3. D. J. Cook and R. M. Hochstrasser, "Intense terahertz pulses by fourwave rectification in air," Opt. Lett. 25(16), 1210-1212 (2000).

4. J. D. B. Clough and X.-C. Zhang, "Laser air photonics: beyond the terahertz gap," Mater. Today 15(1-2), 50-58 (2012).

5. M. D. Thomson, M. Kress, T. Löffler, and H. G. Roskos, "Broadband THz emission from gas plasmas induced by femtosecond optical pulses: From fundamentals to applications," Laser \& Photonics Rev. 1, 349 (2007).

6. I. Babushkin, W. Kuehn, C. Köhler, S. Skupin, L. Berge, K. Reimann, M. Woerner, J. Herrmann, , and T. Elsaesser, "Ultrafast spatiotemporal dynamics of terahertz generation by ionizing two-color femtosecond pulses in gases," Phys. Rev. Lett. 105, 053903 (2010).

7. W. Ji, C.-H. Lee, P. Chen, W. Hu, Y. Ming, L. Zhang, T.-H. Lin, V. Chigrinov, and Y.-Q. Lu, "Meta-q-plate for complex beam shaping," Sci. Reports 6 (2016).

8. P. Stanislovaitis, M. Ivanov, A. Matijosius, V. Smilgevicius, and T. Gertus, "Generation of radially polarized beams and higher order polarization singularities by optical parametric amplification of optical vortices," Opt. Eng. 56, 095101 (2017).

9. J. Durnin, "Exact solutions for nondiffracting beams. i. the scalar theory," J. Opt. Soc. Am. A 4(4), 651-654 (1987).

10. V. Vaicaitis and S. Paulikas, "Formation of bessel beams with continuously variable cone angle," Opt. Quantum Electron. 35(11), 1065-1071 (2003).

11. G. Siviloglou and D. Christodoulides, "Accelerating finite energy airy beams," Opt. Lett. 32(8), 979-981 (2007).

12. S. W. Hell and J. Wichmann, "Breaking the diffraction resolution limit by stimulated emission: stimulated-emission-depletion fluorescence microscopy," Opt. Lett. 19, 780-782 (1994).

13. M. Ivanov and D. Hanstorp, "Controlled spin of a nonbirefringent droplet trapped in an optical vortex beam," Opt. Commun. 427, 152 - 157 (2018).

14. J. He, X. Wang, D. Hu, J. Ye, S. Feng, Q. Kan, and Y. Zhang, "Generation and evolution of the terahertz vortex beam," Opt. Express 21, 20230-20239 (2013).

15. R. Imai, N. Kanda, T. Higuchi, K. Konishi, and M. Kuwata-Gonokami, "Generation of broadband terahertz vortex beams," Opt. Lett. 39, 37143717 (2014).

16. K. Miyamoto, B. J. Kang, W. T. Kim, Y. Sasaki, H. Niinomi, K. Suizu, F. Rotermund, and T. Omatsu, "Highly intense monocycle terahertz vortex generation by utilizing a tsurupica spiral phase plate," Sci. Reports 6, 38880 (2016).

17. S. Ge, P. Chen, Z. Shen, W. Sun, X. Wang, W. Hu, Y. Zhang, and Y. Lu, "Terahertz vortex beam generator based on a photopatterned large birefringence liquid crystal," Opt. Express 25, 12349-12356 (2017).

18. S.-J. Ge, Z.-X. Shen, P. Chen, X. Liang, X.-K. Wang, W. Hu, Y. Zhang, and Y.-Q. Lu, "Generating, separating and polarizing terahertz vortex beams via liquid crystals with gradient-rotation directors," Crystals 10, 314 (2017).

19. A. Minasyan, C. Trovato, J. Degert, E. Freysz, E. Brasselet, and E. Abraham, "Geometric phase shaping of terahertz vortex beams," Opt. Lett. 42, 41-44 (2017).

20. C. Liu, J. Liu, L. Niu, X. Wei, K. Wang, and Z. Yang, "Terahertz circular airy vortex beams," Sci. Reports 7, 3891 (2017).

21. Z. Wu, X. Wang, W. Sun, S. Feng, P. Han, J. Ye, Y. Yu, and Y. Zhang, "Vectorial diffraction properties of thz vortex bessel beams," Opt. Express 26, 1506-1520 (2018).

22. Q. Lin, S. Zheng, Q. Song, X. Zeng, Y. Cai, Y. Li, Z. Chen, L. Zha, $X$. Pan, and S. Xu, "Generation of terahertz vortex pulses without any need of manipulation in the terahertz region," Opt. Lett. 44, 887-890 (2019).
23. A. A. Dhaybi, J. Degert, E. Brasselet, E. Abraham, and E. Freysz, "Terahertz vortex beam generation by infrared vector beam rectification," J. Opt. Soc. Am. B 36, 12-18 (2019).

24. K. Y. Kim, A. J. Taylor, S. L. Chin, and G. Rodriguez, "Coherent control of terahertz supercontinuum generation in ultrafast laser-gas interactions," Nat. Photon. 2, 605 (2008).

25. M. Ammosov, N. Delone, and V. Krainov, "Tunnel ionization of complex atoms and of atomic ions in an alternating electric field," Sov. Phys. JETP 64, 1191 (1986).

26. G. L. Yudin and M. Y. Ivanov, "Nonadiabatic tunnel ionization: Looking inside a laser cycle," Phys. Rev. A 64, 013409 (2001).

27. I. Thiele, B. Zhou, A. Nguyen, E. Smetanina, R. Nuter, K. J. Kaltenecker, P. G. de Alaiza Martínez, J. Déchard, L. Bergé, P. U. Jepsen, and S. Skupin, "Terahertz emission from laser-driven gas plasmas: a plasmonic point of view," Optica 5, 1617-1622 (2018).

28. E. Cabrera-Granado, Y. Chen, I. Babushkin, L. Bergé, and S. Skupin, "Spectral self-action of thz emission from ionizing two-color laser pulses in gases," New J. Phys. 17, 023060 (2015).

29. K.-Y. Kim, J. H. Glownia, A. J. Taylor, and G. Rodriguez, "Terahertz emission from ultrafast ionizing air in symmetry-broken laser fields,' Opt. Express 15, 4577 (2007).

30. M. Gecevicius, M. Ivanov, M. Beresna, A. Matijosius, V. Tamuliene, T. Gertus, A. Cerkauskaite, K. Redeckas, M. Vengris, V. Smilgevicius, and P. G. Kazansky, "Toward the generation of broadband optical vortices: extending the spectral range of a q-plate by polarizationselective filtering," J. Opt. Soc. Am. B 35, 190-196 (2018).

31. P. Klarskov, A. C. Strikwerda, K. Iwaszczuk, and P. U. Jepsen, "Experimental three-dimensional beam profiling and modeling of a terahertz beam generated from a two-color air plasma," New J. Phys. 15, 075012 (2013)

32. V. Vaicaitis, M. Ivanov, K. Adomavicius, Z. Svirskas, U. Morgner, and I. Babushkin, "Influence of laser-preformed plasma on thz wave generation in air by bichromatic laser pulses," Laser Phys. 28, 095402 (2018)

33. V. Denisenko, V. Shvedov, A. S. Desyatnikov, D. N. Neshev, W. Krolikowski, A. Volyar, M. Soskin, and Y. S. Kivshar, "Determination of topological charges of polychromatic optical vortices," Opt. Express 17, 23374-23379 (2009).

34. M. Kolesik and J. V. Moloney, "Nonlinear optical pulse propagation simulation: From Maxwell's to unidirectional equations," Phys. Rev. E 70, 036604 (2004).

35. A. Vinçotte and L. Bergé, "Femtosecond optical vortices in air," Phys. Rev. Lett. 95, 193901 (2005). 